\title{
Automated Inspection using Database Technology within the Aerospace Industry
}

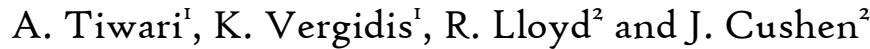 \\ ${ }^{\mathrm{I}}$ Decision Engineering Centre, Manufacturing Department \\ School of Applied Sciences \\ Cranfield University, Cranfield, \\ Bedfordshire, MK43 oAL, U.K. \\ Phone: +44 (o) I234 750 III (ext. 5654) \\ Fax: +44 (o) 1234754605 \\ e-mails: \{a.tiwari, k.vergidis\}@cranfield.ac.uk \\ ${ }^{2}$ Airbus UK Ltd \\ Chester Road, Broughton \\ CHESTER, $\mathrm{CH}_{4}$ oDR, U.K>
}

\begin{abstract}
Many manufacturing processes have become fully automated resulting in high production volumes. However, this is not the case for inspection. Shortening the inspection times in manufacturing industry using the available information resources can result in the reduction of production lead-time and overall costs. Rapid advances in machine tool technology have resulted in fast processing Computer Numerical Control (CNC) machines that are capable of manufacturing parts at high speeds, turning their manual inspection process into a bottleneck. However, many CNC machines record the operations that they perform as Realisation Logs. This paper proposes an approach that utilises these Realisation Logs for automating the inspection process. The automation occurs with the implementation of a software tool that imports and compares the Realisation Logs with the Manufacturing Instructions for a manufactured part. The output of the tool is an Inspection Report that lists all the identified skipped or mishandled operations for that part. The proposed inspection approach is compared with the manual practice within an Aerospace manufacturer. The results demonstrate drastic reduction in production lead-time while producing accurate and reliable Inspection Reports in an automated manner.
\end{abstract}




\section{Introduction}

Increasing levels of automation in manufacturing industry have not yet led to similar levels of automation in the inspection of intermediate and finished products. Therefore, it is a continuous challenge for the Inspection Engineers to cope with the pace of high speed automatic CNC machines, to ensure inspection quality in comparable time frames. One solution has been the use of highly automated machines to carry out inspection such as robots, scanning or artificial visionary devices, but this is an expensive solution not always practical. It also ignores the vast data produced by modern CNC machines as they perform their tasks. Contemporary CNC machines in aerospace industry such as the Electro-Impact machines are used for a variety of purposes including wing manufacturing and final assembly. These fully automated machines operate uninterrupted at high-speed and record operational information continuously in the form of logs. This paper proposes an approach based on the utilisation of these logs for automating the inspection process The proposed approach is focused on identifying skipped and mishandled manufacturing operations based on the data fed and recorded by the CNC machines.

\section{Related work}

In the past, many developments have been made to enhance the efficiency of manufacturing operations as well as the quality of inspection along the lifecycle of products. This section examines the recent trends in manufacturing inspection processes and the possible links with database technology.

Although inspection plays a vital role in quality control and plant maintenance, according to Bosserman (2006), manufacturers prefer to allocate funds on new manufacturing equipments rather than invest in development of new technology for inspection of products. The development for a complete system of inspection and quality control of manufactured parts requires the coordination of a set of complex processes that they allow data acquisition, dimensional evaluation and comparison with a reference process model (Boukebbab et al., 2007). Some manufacturers even consider outsourcing the inspection of their products, expecting to benefit from the advanced technologies in product inspection (Bosserman, 2006). Lee (2006) proposed a model for investment in inspection and the investment in measurement equipment as these two affect warranty cost, market increase, and costs. 
In terms of specific approaches, most of the advances in automated inspection originate from the aerospace and automotive industries. There are many advances in automated quality inspections using high technology equipment such as robots, artificial visual inspections based on X-rays, laser digitising system and some non-destructive inspections using Eddy current method (Siegel, 1994). Kuhlenkotter and Sdahl (2007) propose an automated approach for the inspection process that utilises robot programming in order to increase inspection quality and decrease production costs. Colet (2006) discusses the development of machine vision as a fast and reliable tool for quality control inspection. The application of computer vision to industry and manufacturing can aid manufacturing organisations in reaching their quality goals. Colet (2006) concludes that many industries are beginning to adopt universal quality inspection with cost effective machine vision systems for optimum quality. O'Dell (200I) proposes an automated system to inspect the O-ring sealing surfaces on the Space Shuttle Reusable Solid Rocket Motor (RSRM) case segment joints. A laser digitising system is used to create a $3 \mathrm{D}$ map of the $\mathrm{O}$-ring sealing surfaces which are further analysed and inspected for any irregularities. Another example is the Printed Circuit Board (PCB) assembly process in which an automated solder joint visual inspection method is introduced after development of the surface mount technology (Oyeleye, 1999).

There are some papers about the development of automatic inspection point placement and path planning methods, for use in the integration of Computer-Aided Design (CAD) with Coordinate Measuring Machine (CMM) inspection (Albuquerque et al., 200o). Three-dimensional shape measurements could also be carried out using optical methods focussing on structured light techniques (Chen et al. 2000). Prieto et al. (2002) propose an automated inspection system for a manufactured part using a cloud of $3 \mathrm{~d}$ measured points -provided by a range sensor- and its CAD model. In spite of the high precision attained by Coordinate Measuring Machines (CMM), range sensors offer significant advantages for dimensional inspection such as a high speed of digitisation and the capacity to take $3 \mathrm{~d}$ measurements on the whole surfaces of a part without physical contact. Also, Killmaier and Babu (2003) developed a method to detect form deviations of standard geometrical features (line, circle, plane, cylinder, cone and sphere) of a manufactured part using a Genetic Algorithm (GA). The GA arrives at the optimal values of these basic deviation types which reproduce the profile very close to the measured one. 
There are a significant number of papers in the area of automated quality inspection using high technology equipment but there is a lack of research in automated inspection based on the production data available. Apart from utilising high technology equipment to facilitate the inspection process, CNC machine output can be also used. However, there is no reported work that exploits database technology in order to enhance the inspection process. This paper discusses the utilisation of the machine realisation logs and their comparison with the manufacturing instructions. This proposed approach promotes utilisation of resources and data available to further enhance the efficiency and automation of the inspection process. In order to achieve this, database technology is utilised to transform and normalise the available data. This is a new approach to automate the inspection process without involving capital investment or introducing new equipment.

\section{Wing manufacturing and inspection process}

This section presents the manufacturing context of the proposed inspection approach. This approach was developed within a particular aerospace company and it was designed to tackle the long inspection times of inspecting manufactured aircraft wings. This poses a challenge due to the fact that wing manufacturing is a complex process. Moreover, the requirements of the end result must meet the highest standards of quality and safety due to the nature of the product. This section also briefly describes the concept of inspection within the given context.

Aircraft wings are shaped by panels that provide the strength and structure to the wings. Figure I demonstrates the basic structure of an aircraft wing. Each wing contains up to ten panels of different lengths and sizes. The manufacturing process for these panels involves drilling, riveting, bolting, cold working and fastening of around 26,0oo holes on these panels. These operations are performed by Electro-Impact CNC machines working at high speeds. These machines record all the operations that they perform in the form of realisation logs that are continuously generated for each panel. As the machines are working uninterrupted, the realisation logs are generated during execution. These logs not only record the operations performed by the machine but also store information about a range of parameters related to the operation (e.g. precision achieved, voltages, etc.). One machine can work upon different panels in a sequence, and one panel can pass through 
different machines before it is finalised. A key challenge is to extract the information regarding the operations performed on a single panel from the logs of various machines that operate continuously on different panels.

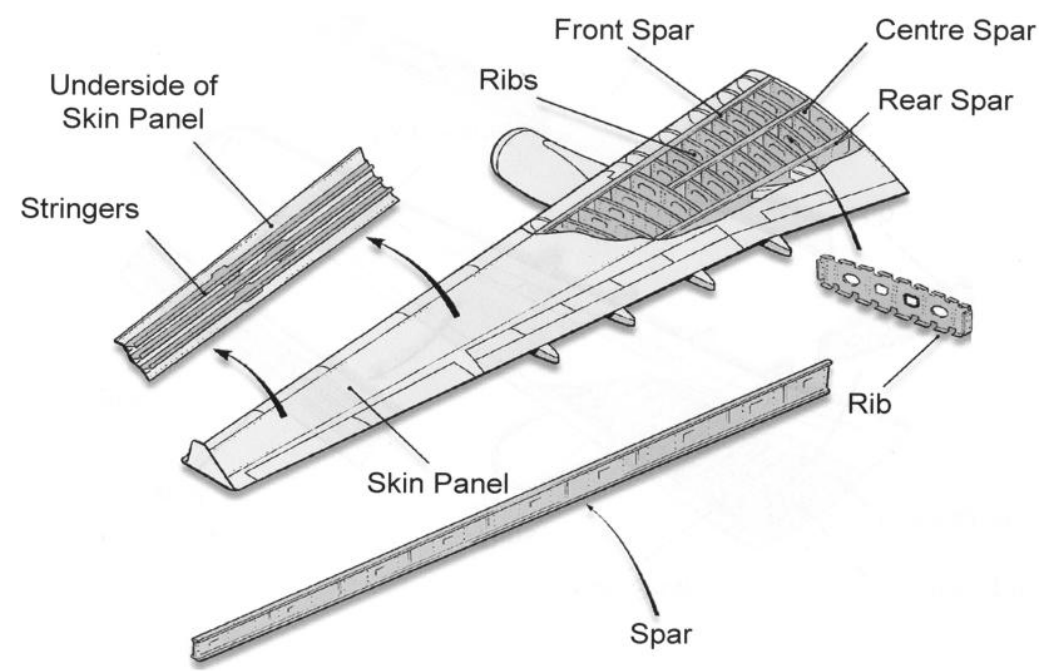

Figure I. Basic structure of an aircraft wing

The inspection process involves the manual inspection of each manufactured wing panel for two main types of errors: (i) missing holes and (ii) missing fasteners. Manual inspection can be very time consuming with inspection duration for a wing set up to 120 hours. This is due to the fact that the inspectors check one by one all the holes and fasteners in every single wing panel in order to locate any missing operations or damage. This results in inspection potentially being the bottleneck of a fast manufacturing process along with the quality issues. What is needed is to automate the inspection process by automatically locating the erroneous operations based on predefined criteria. This not only tackles the long inspection times but can also ensure that all erroneous operations are located in a consistent and trustworthy fashion. There would still remain a requirement to manually inspect, at a reduced level in order to check for damage. However, checking the correlation of the installed fasteners to design intent would no longer be required post First Article Inspection Report (FAIR) (First part must be checked against design intent for proof of concept and validation of NC data). The next section discusses our proposed approach in order to address the issues that arise in manual inspection. 


\section{Proposed inspection approach}

This section describes the authors' approach for the automation of the inspection process. This approach consists of two main steps: (i) the development of an algorithm that automates the inspection process using data from the manufacturing operations; and (ii) the implementation of this algorithm in a software that suits the requirements of the inspector engineers and contributes to improving the inspection process by reducing the time needed to inspect a panel and increasing the quality and consistency of inspection results.

\section{I The comparison algorithm}

The proposed approach utilises the data from two different databases. The first database is named Manufacturing Instructions $\mathrm{Db}$ (MIDb) and it contains the data that are used as inputs for the CNC machines. It contains the instructions for all the operations that the machine is expected to perform. The second database is called Realisation Logs $D b(R L D b)$ and it contains the output $(\log )$ of the machines. It records the data about the operations that the machine has actually performed. The proposed approach is based on the concept that by comparing the input (instructed operations) and the output (performed operations) of the machines, the operations that were mishandled or omitted can be identified in a timely fashion.

This can be realised by comparing the data stored in these two databases (MIDb and $\mathrm{RLDb})$. Any difference in the data recorded in these two databases is recorded as an error. Two types of errors are of interest: (a) skipped operations, i.e. manufacturing operations that the machine did not perform and (b) mishandled operations, i.e. manufacturing operations that the machine performed but not as instructed or were incomplete. In the first case of errors, the operation details are stored in the MIDb but they are not recorded in the RLDb. In the second case, the operation details are stored in both databases but in $\mathrm{RLDb}$ some information is altered based on how the operation was actually performed. It important to note that $\mathrm{MIDb}$ is $100 \%$ reliable as it contains the validated manufacturing instructions for all the operations of the CNC machines. These instructions are validated by the manufacturing department of the aerospace manufacturer before they are sent to the CNC machines and they are confirmed by FAIR. Also, the output of the CNC machines is considered fully reliable as the machines accurately record each operation that 
they perform on the panel. This is confirmed by the machine manufacturer in the machine manual.

Figure 2 demonstrates the main steps in the comparison. The main inputs for the comparison are the data stored in each of the two databases. Before the actual comparison the data contained in the databases need to be normalised. This is due to the fact that CNC machines produce the logs in the form of text files. These text files in the RLDb need to be re-structured following the schema (field names, tables, etc.) of the MIDb so that direct comparison can take place. Comparing the databases and identifying any discrepancies results in two types of errors discussed previously: skipped and mishandled operations. A list of these types of errors is compiled in an inspection report that provides a coherent reference of the operations that were either omitted or performed in an inaccurate fashion.

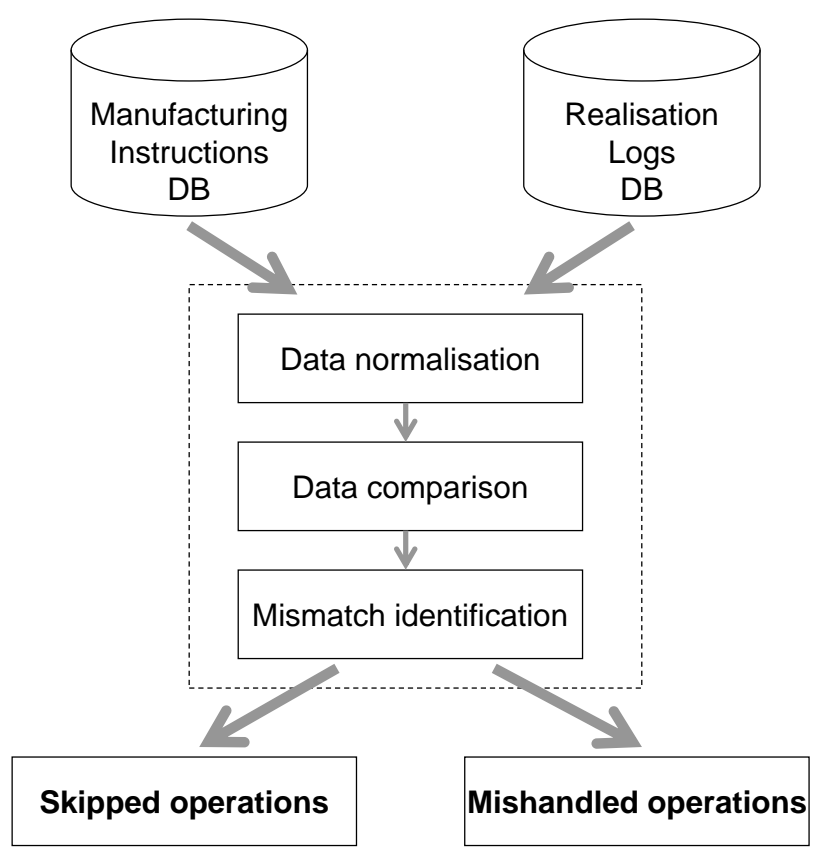

Figure 2. Main steps of database comparison for identifying erroneous operations

Figure 3 presents a more detailed view of our approach by demonstrating in a flowchart the main steps of the comparison algorithm. Once the databases are in the same format, each operation goes through the comparison algorithm. The aim of the algorithm is to compare the manufacturing operations as they are recorded and stored in the two databases and identify the erroneous ones. The fundamental criteria for comparison are the $\mathrm{x}, \mathrm{y}, \mathrm{z}$ coordinates of an operation. In addition to these, other parameters such as voltage, current, 
spindle speed or feed, spindle position, A/B axis position can be used as criteria for error detection. These two categories of criteria are essential in order to identify the operations that are skipped and incorrectly performed by the CNC machines.

As shown in figure 3, the comparison algorithm uses as inputs the data stored in MIDb about the operation instructions and the data recorded in RLDb regarding the operations performed. The steps in the dotted box occur for every operation that is stored in MIDb. At first, each operation is evaluated based on its instructed coordinates (XYZ) and its recorded coordinates ( $\mathrm{X}^{\prime} \mathrm{Y}^{\prime} \mathrm{Z}^{\prime}$ ). The instructed coordinates originate from the MIDb and the recorded coordinates from the RLDb as these are the outputs of the CNC machines. If an operation from MIDb with XYZ coordinates does not have a corresponding match in $\mathrm{RLDb}$, then the operation is considered as skipped. In order to separate skipped from mishandled operations, there is a predefined tolerance level. If an operation is performed within this level, it is considered as mishandled, whereas if it is outside this level it is considered as skipped. Mishandled operations are identified by comparisons using other criteria such as voltages, stack value, fastener length and currents. If these criteria match in terms of instructed and performed details then the operation is considered successful.

This process is repeated for all the operations stored in the Manufacturing Instructions database. Once this process is completed the Inspection Report is generated. This report contains all the operations that the algorithm has flagged as erroneous. Each operation is listed as skipped or mishandled. In the case of mishandled operations, further details about the error are provided (e.g. the criteria that fell outside acceptable tolerances). The generation of this report can contribute to determining the quality of a manufacturing process in an accurate and timely fashion while reducing the necessity for manual inspection of each operation performed. 


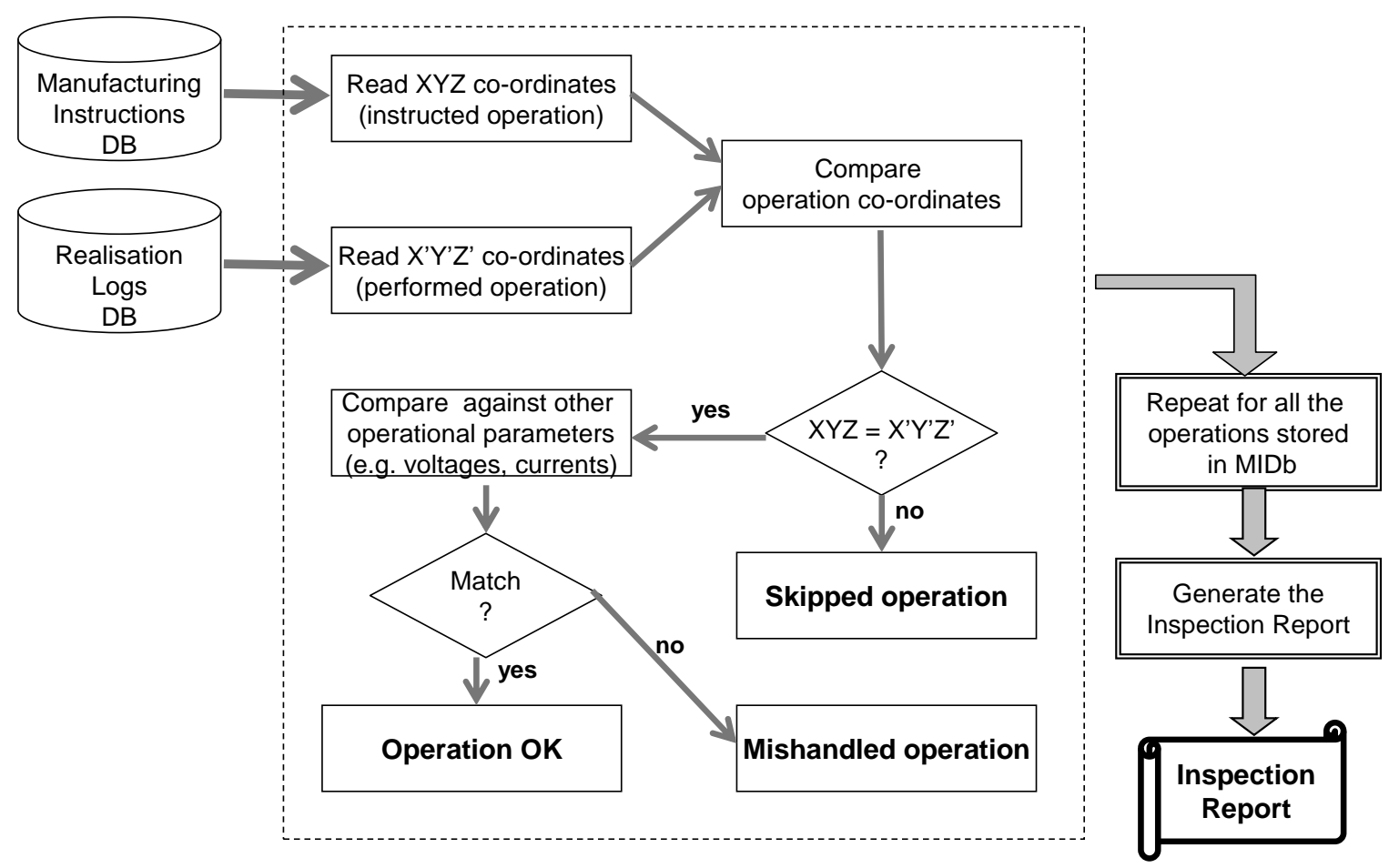

Figure 3. Generation of the Inspection Report by the comparison algorithm

\subsection{Development of an automated inspection tool}

The second step of the approach for the automation of the inspection process involves the implementation of a software application based on the comparison algorithm described in the previous section. The application normalises and compares the data from MIDb and $\mathrm{RLDb}$, and generates a variety of customised Inspection Reports in an automated manner. The scope of the application is to automate the inspection for a range of wing panels. The main steps for the implementation of the inspection tool involve:

I. Investigation of the structure and form of the data required.

2. Transforming noisy and redundant data into useful information.

3. Designing the schemas of the two databases in order to utilise data normalisation and direct comparison.

4. Extracting information for a specific panel from different data sources.

5. Development of the comparison algorithm to compare the panel information contained in the two databases.

6. Implementation of the above functionalities with a simple user interface.

7. Presentation of the results 
The software tool is developed in Microsoft Access utilising Visual Basic elements (such as forms, macros, etc.) and SQL queries in order to generate the automatic inspection reports. The queries contribute to locating and comparing relevant data from the databases and the Visual Basic elements help in creating a user-friendly interface. The enormity and complexity of databases pose a challenge for the normalisation process as each of the databases contains on average more than 200,000 records. The databases are in different formats without clear structure containing fragmented information. The Manufacturing Instructions in their initial form come in textual or Microsoft Excel format and Realisation Logs come in textual form. The normalisation process involves the import and conversion of these two formats to the corresponding databases in Microsoft Access.

A snapshot of the application's user interface is shown in figure 4. The application consists of a series of tabs. These tabs are:

I. Panel selection tab, which provides the opportunity to select a part -a panel in this caseand start automatic inspection process. This is challenging because one machine works upon different panels and one panel goes to different machines before it is finalised. When a specific panel is selected, the software imports all the data about that panel and convert them into the correct format. As the user selects a panel, the associated part number assigned for its identification is selected automatically. The default setting is the selection of last machined panel. Once the required panel is selected and all the fields are completed, the data can be imported and normalised. This occurs by using literal descriptions of the product in the GUI and automatically linking them to the relevant part number via an association table and assists in shop-floor comprehension and acceptance

2. Shop floor Snag-sheet tab, which provides options for a customised generation of the Inspection Report for skipped operations. 'Snag sheet' is how inspection reports are called within the particular aerospace context. As discussed earlier, the identification of skipped operations is carried out by matching the coordinates of each operation recorded in $\mathrm{MIDb}$ with the data in RLDb. Options are provided to generate reports for missing holes and/or missing fasteners -the two main time consuming activities when inspected manually. 


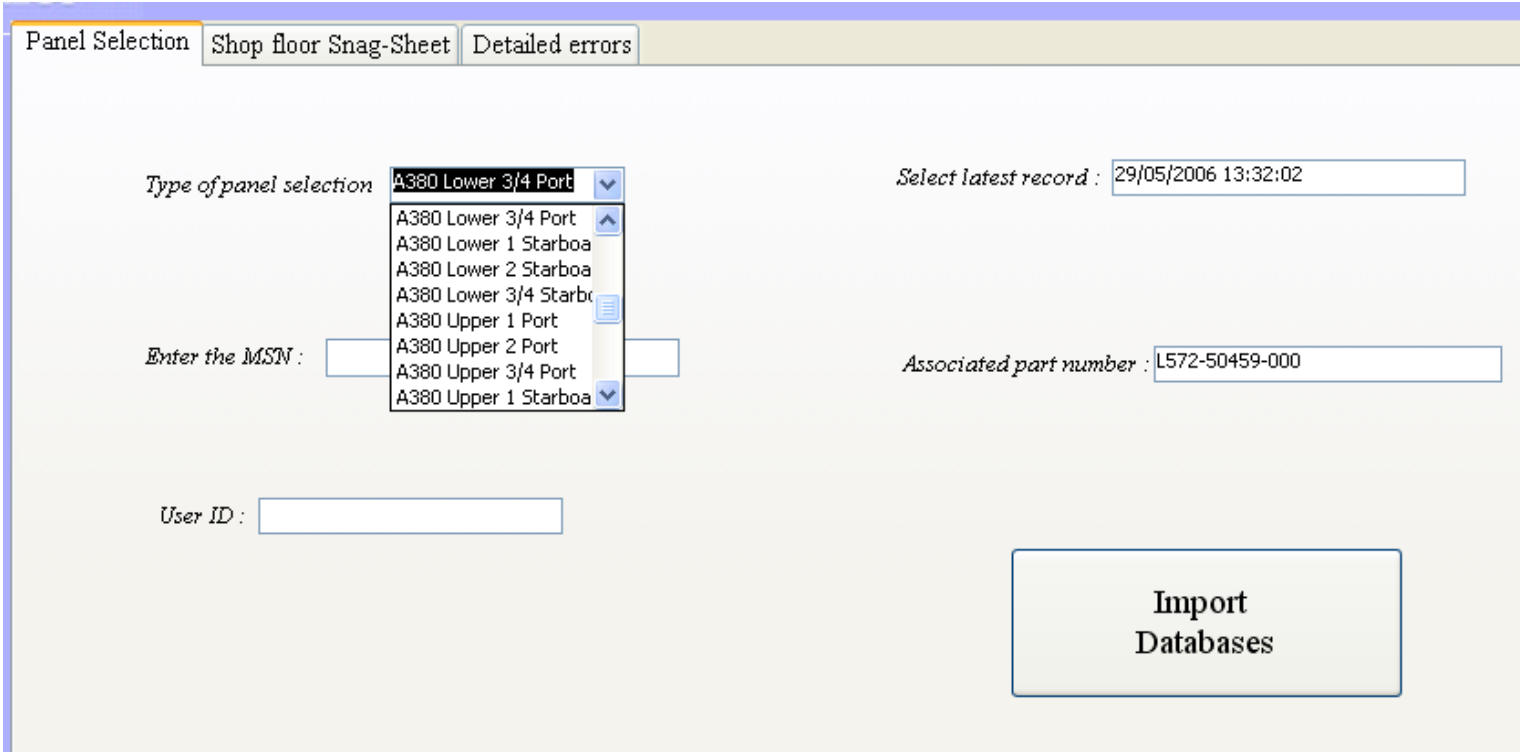

Figure 4. Snapshot of the user interface

3. Detailed errors tab, which provides a list of other types of errors (i.e. mishandled operations) to be included in the Inspection Report. This tab provides the opportunity of selecting an additional and more specific type of error. Examples of manufacturing parameters that could be analysed for detecting possible errors are voltages, stack value, incorrect fastener length and cold work. As discussed earlier, these errors are detected by matching the relevant parameters of the operations stored in MIDb with those recorded in RLDb. The output of any of such comparisons can be visualised in the form of an Inspection Report.

Figures 5 and 6 present instances of such Inspection Reports. Figure 5 demonstrates a list of missing holes (skipped operations) for a specific panel. Each hole is identified by a unique ID that was allocated during the design stage of the panel. For each hole that has been identified as missed, further information is provided such as coordinates, rib number, position, fastener type and diameter to assist in physical location and rectification of the fault. Figure 6 demonstrates an Inspection Report for wrong fastener length (specific type of mishandled operation) for a panel. Again for the operations that have been identified as erroneous based on the specific criterion, detailed information is provided. 


\begin{tabular}{|c|c|c|c|c|c|c|c|c|c|}
\hline \multirow{3}{*}{$\begin{array}{l}\text { PART No. } 52415 \\
\text { Type of Panel: LP2 }\end{array}$} & \multirow{3}{*}{\multicolumn{7}{|c|}{$\begin{array}{l}\text { Inspection Report for: } \\
\text { Missing Holes }\end{array}$}} & \multirow{2}{*}{\multicolumn{2}{|c|}{ User: 74125}} \\
\hline & & & & & & & & & \\
\hline & & & & & & & & Date: $30 /$ & $3 / 2007$ \\
\hline Design Intent ID & PP_X & PP_Y & PP\# & String & Fwd / Aft & Rib No & Position & $\begin{array}{l}\text { Fastener } \\
\text { Type }\end{array}$ & Diameter \\
\hline TST26A22047BF06XBIM & 13824.9 & 17608.2 & 67382 & STR26 & Fwd & 22 & Rib & B & 06 \\
\hline TST26A22054BA06XBIS & 13904 & 17612.8 & 67383 & STR26 & Fwd & 22 & Rib & B & 06 \\
\hline TST26A22055BA06XBIM & 13857 & 17769.9 & 67383 & STR26 & Aft & 22 & Rib & B & 06 \\
\hline
\end{tabular}

Figure 5. Inspection report for skipped operations (missing holes)

\begin{tabular}{|c|c|c|c|c|c|}
\hline MSN: 12365 & \multirow{3}{*}{\multicolumn{4}{|c|}{$\begin{array}{l}\text { Inspection Report for: } \\
\text { Wrong Fasteners Length }\end{array}$}} & \\
\hline PART No. 52415 & & & & & User: 74125 \\
\hline Type of Panel: LP2 & & & & & Date: 30/03/2007 \\
\hline ID & PP_Num & Block_Num & $\begin{array}{l}\text { Commanded } \\
\text { Fastener Length }\end{array}$ & $\begin{array}{c}\text { Installed } \\
\text { Fastener Length }\end{array}$ & $\begin{array}{c}\text { Error in } \\
\text { Fastener Length }\end{array}$ \\
\hline BST21-A34XRTM & 67341 & 139 & 11 & 09 & \\
\hline BST21-A34XRTM & 67341 & 1617 & 20 & 17 & \\
\hline BST21-A34XRTM & 67341 & 1696 & 15 & 13 & \\
\hline
\end{tabular}

Figure 6. Inspection report for other types of errors (wrong fastener length)

\section{Comparison of manual vs. automated inspection}

This section presents the effects of the proposed approach to the inspection process in terms of time and quality improvements. It also provides a comparison of the proposed automated inspection approach with the manual inspection practice that is currently followed within a large aerospace manufacturer. Table i describes the basic elements of the inspection process when performed manually and when it follows the automated approach that this paper proposes.

Table I shows how the current manual practice of inspection can be improved by the proposed software-assisted approach. In the manual approach the most time-consuming steps are 6 and 7 where the Inspection Engineers have to manually inspect every single operation performed on each wing panel and record deviances on an inspection report. This approach also raises issues about the quality of inspection performed, i.e. the 
consistency of report formats and textual information contained therein. Our approach aims at incorporating the software tool as part of the inspection process in order to partly automate it and reduce the need for manual inspection of the operations performed. However, our approach only checks for skipped or mishandled operations performed by the machine. It does not check for other defects, such as those arising due to transportation, fixtures, etc. This means that visual inspection is not eliminated but it is greatly reduced since the manual inspection of the operations performed by the CNC machines is no longer required.

\begin{tabular}{|c|c|c|}
\hline no. & Manual inspection & Automated inspection \\
\hline I & \multicolumn{2}{|c|}{ Design Instructions are passed to Manufacturing department. } \\
\hline 2 & \multicolumn{2}{|c|}{ Generation of Manufacturing Instructions in the form of NC codes. } \\
\hline 3 & \multicolumn{2}{|c|}{ NC codes fed to the CNC machines. } \\
\hline 4 & \multicolumn{2}{|c|}{$\begin{array}{l}\text { CNC machines perform the operations and record them in the } \\
\text { Realisation Logs. }\end{array}$} \\
\hline 5 & $\begin{array}{l}\text { Realisation Logs not further } \\
\text { utilised. }\end{array}$ & $\begin{array}{l}\text { Realisation Logs are normalised } \\
\text { and fed to the comparison } \\
\text { algorithm. }\end{array}$ \\
\hline 6 & $\begin{array}{l}\text { Shop floor inspectors inspect the } \\
\text { panels manually, in association } \\
\text { with drawings, to identify the } \\
\text { skipped operations. }\end{array}$ & $\begin{array}{l}\text { Manufacturing Instructions are } \\
\text { normalised and compared with } \\
\text { Realisation Logs to identify } \\
\text { skipped or mishandled operations. }\end{array}$ \\
\hline 7 & $\begin{array}{l}\text { Inspection Engineers record the } \\
\text { errors manually in reports. }\end{array}$ & $\begin{array}{l}\text { Inspection Reports are generated } \\
\text { automatically by the software tool. }\end{array}$ \\
\hline 8 & $\begin{array}{l}\text { The reports are returned to } \\
\text { Manufacturing department for } \\
\text { undertaking corrective steps. }\end{array}$ & $\begin{array}{l}\text { Rework tasks that are possible on } \\
\text { CNC machines are completed } \\
\text { immediately. }\end{array}$ \\
\hline 9 & $\begin{array}{l}\text { Decision is made on operation-by- } \\
\text { operation basis on whether the } \\
\text { rework will be performed manually } \\
\text { or by CNC machines. Rectification } \\
\text { is generally via manual means as, } \\
\text { due to the inherent time taken to } \\
\text { perform the inspection operation. } \\
\text { Inspection is completed away from } \\
\text { the m/c in order to free the facility } \\
\text { for further production. Manually } \\
\text { installed fasteners are usually } \\
\text { alternatives that require } \\
\text { concessionary reporting }\end{array}$ & $\begin{array}{l}\text { Remaining rework is undertaken } \\
\text { manually. }\end{array}$ \\
\hline Io & $\begin{array}{l}\text { Time consuming repeated } \\
\text { backward loops of rework. }\end{array}$ & $\begin{array}{l}\text { Reduction in backward loops of } \\
\text { rework. }\end{array}$ \\
\hline
\end{tabular}

Table I. Comparison of manual vs. automated inspection process 
In order to demonstrate the time efficiency of the proposed automated inspection, it was incorporated in the inspection process of wing panels for three different aircraft models ( $\mathrm{A}, \mathrm{B}$ and $\mathrm{C})$. The difference between these models is in the quantity of data and the number of operations performed in their wing panels. Testing the tool with different inspection requirements can provide a good indication of the effect it can have on the inspection process. The duration of the inspection process with the tool was measured and compared with the duration of the manual inspection process. The results obtained demonstrate that the software tool provides a drastic reduction in the time required to inspect the panels.

Table 2 demonstrates the results for the aircraft model $\mathrm{A}$. The manual inspection takes up to 120 hours without the capability for checking other types of quality errors. The proposed approach is capable of producing the Inspection Reports in 6 hours, performing not only the missed operation inspection, but also more quality checks, in $5 \%$ of original time. The results for aircraft model B in table 3 concern a wing set smaller than A. Again there is a $95 \%$ reduction in the duration of the inspection process. The manual approach takes 6o hours per wing set as opposed to 3 hours for the automated approach. The aircraft model $\mathrm{C}$ contains the smallest wing set that requires an $\mathrm{i} 8$-hour manual inspection. The automated approach requires less than an hour, providing a $94 \%$ reduction in the inspection time required. It is important to note that the data for each model of aircraft were provided in different formats. The software tool was able to import and normalise the data as it supports inputs in a variety of formats (.txt, .db, .xls, etc).

\begin{tabular}{|c|l|c|c|}
\hline no. & \multicolumn{1}{|c|}{$\begin{array}{c}\text { Inspection \& } \\
\text { Report compilation of: }\end{array}$} & $\begin{array}{c}\text { Manual inspection } \\
\text { (hours) }\end{array}$ & $\begin{array}{c}\text { Software-assisted } \\
\text { inspection } \\
\text { (hours) }\end{array}$ \\
\hline I & Missing holes & 60 & 2 \\
\hline 2 & Missing fasteners & 30 & 0.5 \\
\hline 3 & Mishandled operations & 30 & 0.5 \\
\hline 4 & Other types of errors & - & 3 \\
\hline & $\begin{array}{l}\text { TOTAL DURATION: } \\
\text { (for a wing set) }\end{array}$ & $\mathbf{1 2 0}$ & 6 \\
\hline
\end{tabular}

Table 2. Manual vs. Software-assisted inspection for aircraft model A 


\begin{tabular}{|c|l|c|c|}
\hline no. & \multicolumn{1}{|c|}{$\begin{array}{c}\text { Inspection \& } \\
\text { Report compilation of: }\end{array}$} & $\begin{array}{c}\text { Manual inspection } \\
\text { (hours) }\end{array}$ & $\begin{array}{c}\text { Software-assisted } \\
\text { inspection } \\
\text { (hours) }\end{array}$ \\
\hline I & Missing holes & 30 & I \\
\hline 2 & Missing fasteners & I5 & 0.25 \\
\hline 3 & Mishandled operations & I5 & 0.25 \\
\hline 4 & Other types of errors & - & I.5 \\
\hline & $\begin{array}{l}\text { TOTAL DURATION: } \\
\text { (for a wing set) }\end{array}$ & 60 & 3 \\
\hline
\end{tabular}

Table 3. Manual vs. Software-assisted inspection for aircraft model B

\begin{tabular}{|c|l|c|c|}
\hline no. & $\begin{array}{c}\text { Inspection \& } \\
\text { Report compilation of: }\end{array}$ & $\begin{array}{c}\text { Manual inspection } \\
\text { (hours) }\end{array}$ & $\begin{array}{c}\text { Software-assisted } \\
\text { inspection } \\
\text { (hours) }\end{array}$ \\
\hline I & Missing holes & 9 & 0.3 \\
\hline 2 & Missing fasteners & 4.5 & 0.08 \\
\hline 3 & Mishandled operations & 4.5 & 0.08 \\
\hline 4 & Other types of errors & - & 0.4 \\
\hline & $\begin{array}{l}\text { TOTAL DURATION: } \\
\text { (for a wing set) }\end{array}$ & $\mathbf{1 8}$ & $<\mathrm{I}$ \\
\hline
\end{tabular}

Table 4. Manual vs. Software-assisted inspection for aircraft model C

The proposed automated inspection approach generates the reports automatically with no human intervention. According to the Aerospace manufacturer, the accuracy of the manually produced reports during the first inspection was estimated to be around $99.9 \%$. As each panel has 26,000 holes the estimated error in reporting is 26 operations per panel. The inspection operation is performed a second time, following rectification of the original faults, thus 100\% accuracy is guaranteed at the expense of time. The automated inspection approach is reported to have 100\% accuracy identifying correctly all the operations that fall in the category of skipped or mishandled; also eliminating the need for a second round of manual inspection. Therefore, the inspection reports with the proposed automated approach are more accurate, reliable and efficient in terms of time.

Also the presentation of the report is improved making the reports easy to read and understand. Each Inspection Report is tailored for different kinds of errors providing the most suitable information in each case. The information that appears in each report is decided in conjunction with Inspection Engineers and their requirements. An important advantage of the automated approach is that it can be used at run time with the machines. 
As the Realisation Logs are generated, they are normalised and compared with the Manufacturing Instructions in order to determine the quality of the operations performed.

The outcome of the software tool was compared with the results from the relevant manual inspection process, and also independently tested and validated by the Quality department of the Aerospace manufacturer. The results were found to be more accurate than those from the relevant manual inspection processes due to the removal of human errors. The accuracy and reliability of both the inputs (Manufacturing instructions and realisation logs) were also validated. However, as demonstrated by the results, the software-assisted inspection was performed in a considerably reduced time.

\section{Discussion}

This section highlights the benefits of the proposed inspection approach as well as its limitations. It concludes by providing directions for future research. The work presented in this paper investigates the utilisation of the input and output data of CNC manufacturing machines for automating the inspection process. To the authors' knowledge the output of the CNC machines is not used in any previous automated inspection approach. Normalising the Realisation Logs and converting them to a database with identical schema as the Manufacturing Instructions creates a novel approach for automating the inspection process without investing in expensive automation technology. This paper makes a first step towards a new perception of inspection automation in manufacturing industry.

The benefits of this approach, as highlighted by the comparison with the manual inspection practice, are two-fold: (i) the drastic reduction in the inspection duration and (ii) the reliability and confidence in the quality of the inspection results. The reduction in inspection duration implies the removal of routine and tedious manual inspection of every single manufacturing operation by exploiting the output of the CNC machines. The proposed approach can be applied to any manufacturing process that shares the same characteristics with the process that this paper described. Essentially, if a manufacturing process has a stored input and output that can be normalised and compared, then the proposed approach can be applied. 
One of the limitations of the proposed approach lies in the assumption that when the CNC machines perform an operation, they record it in the Realisation Logs in a reliable manner. However, in the case of some controller malfunctions, the machine does not produce an accurate output and this possibility needs to be taken into account. Another limitation of the proposed inspection approach is that it is not capable of handling inspection issues that are not linked with the CNC operations such as damage caused by handling and/or fixtures.

Future work can involve the real-time execution of the inspection tool while a part is manufactured. This will provide the capability of detecting errors as they occur, thus the decision making process of rework can be shortened and made more effective. Also, the proposed approach could be further supported by soft computing techniques and modern information system methods in order to develop knowledge-based decision support systems. Soft computing techniques exploit the tolerance for uncertainty and approximate reasoning for making decisions in real-life ambiguous environments. Intelligent analysis of the parameters could be carried out to avoid errors. The decisions about whether the rework has to be done manually or by a machine could also be taken by these intelligent techniques. The manual inspection for detecting physical errors such as bends, breaks etc. could also be carried out by monitoring the parameters. When the tool breaks or damages the part, the parameters change abruptly and this is shown in realisation logs. Those specific operations or part of an operation that have a sudden change of parameters could also be identified by the proposed approach in the Inspection Report. Also, the potential of drilling into historical data to locate consistent or repeated problems is appealing using the proposed approach. Finally, the proposed philosophy could be extended to other parts and industries that use modern CNC machines in manufacturing and assembly.

\section{Conclusions}

This paper proposed a novel approach for automating the inspection process of a manufactured part by utilising the data available. This data are stored in two main databases: the Manufacturing Instructions, which contain the operations instructed to the machines and the Realisation Logs, which contain the operations performed by the machines. The comparison of these two databases helps in identifying any skipped or mishandled operations. The proposed approach for automated inspection was compared 
with the manual practice and the results indicate a strong reduction in inspection leadtime for a part. Moreover, the inspection reports are more reliable and accurate in terms of quality output. The proposed approach also reduces manual rework, improves CNC machine utilisation and identifies errors that are difficult to be detected manually. The results demonstrated in this paper are encouraging for further research in the area of industrial automation through database technology.

\section{References}

Albuquerque V. A., Liou F. W. and Mitchell O. R. (2000) 'Inspection point placement and path planning algorithms for automatic CMM inspection'. International Journal of Computer Integrated Manufacturing, vol. 13, pp. 107-120.

Bosserman D. (2006) 'Should you outsource inspection?', Manufacturing Engineering 137 (5), pp.r8.

Boukebbab S., Bouchenitfa H, Boughouas H. and Linares J.M. (2007) 'Applied iterative closest point algorithm to automated inspection of gear box tooth', Computers and Industrial Engineering, 52 (I), pp. 162-173.

Chen F., Gordon M. and Brown M. S. (200o) 'Overview of three-dimensional shape measurement using optical methods'. Optical Engineering, vol. 39, pp. I0-22.

Colet P. (2006) 'Machine vision aids quality inspection', Quality 45 (10), pp. 22-23

Killmaier T. and Babu R. A. (2003) 'A genetic approach for automatic detection of form deviations of geometrical features for effective measurement strategy'. Precision Engineering, vol. 27, pp. 370-38I.

Kuhlenkotter B. and Sdahl M. (2007) 'Automated inspection system for headlamp reflectors'. International Journal of Advanced Manufacturing Technology 32, pp. 500-504.

Lee H-H. (2006) 'Investment model development for repetitive inspections and measurement equipment in imperfect production systems', International Journal of Advanced Manufacturing Technology 31, pp. 278-282. 
O'Dell K.P. (200I) 'Automated inspection of the RSRM case O-ring seal surface [Reusable Solid Rocket Motor]'. In Proceedings of the 37th Joint Propulsion Conference and Exhibit by the American Institute of Aeronautics \& Astronautics UT, 8-II July 200I, Salt Lake City, United States.

Oyeleye O. (1999) 'Automatic visual inspection of surface mount solder joint defects'. International Journal of Production Research, vol. 37, pp. 1217-1242.

Prieto F., Redarce T., Lepage R. and Boulanger P. (2002) 'An Automated Inspection System’. International Journal of Advanced Manufacturing Technology, vol. 19, pp. 917-925.

Siegel, M.W. (1994) 'Automation for non-destructive inspection of aircraft'. In Proceeding of the conference on Intelligent Robots in Field, Factory, Service and Space (CIRFFSS '94), Houston, Texas, pp. 367-377. 